\title{
Nanopumping Molecules via a Carbon Nanotube
}

\author{
Min Chen ${ }^{1,2,3}$, Ji Zang ${ }^{3}$, Dingquan Xiao ${ }^{1}$, C. Zhang ${ }^{4}$, and Feng Liu ${ }^{3}(\bowtie)$ \\ ${ }^{1}$ Department of Materials Science and Engineering, Sichuan University, Chengdu, Sichuan 610064, China \\ ${ }^{2}$ Department of Optoelectronic Technology, Chengdu University of Information Technology, Chengdu, Sichuan 610225, China \\ ${ }^{3}$ Department of Materials Science and Engineering, University of Utah, Salt Lake City, Utah 84112, USA \\ ${ }^{4}$ School of Engineering Physics, University of Wollongong, New South Wales 2522, Australia
}

Received: 1 September 2009 / Revised: 29 September 2009 / Accepted: 17 October 2009

OTsinghua University Press and Springer-Verlag 2009. This article is published with open access at Springerlink.com

\begin{abstract}
We demonstrate the feasibility of using a carbon nanotube to nanopump molecules. Molecular dynamics simulations show that the transport and ejection of a $C_{20}$ molecule via a single-walled carbon nanotube (SWNT) can be achieved by a sustained mechanical actuation driven by two oscillating tips. The optimal condition for nanopumping is found when the tip oscillation frequency and magnitude correlate to form quasi steady-state mechanical wave propagation in the SWNT, so that the energy transfer process is optimal leading to maximal molecular translational motion and minimal rotational motion. Our finding provides a potentially useful mechanism for using an SWNT as a vehicle to deliver large drug molecules.
\end{abstract}

\section{KEYWORDS}

Carbon nanotube, nanopumping, drug delivery, MD simualtion

\section{Introduction}

Some important applications of carbon nanotubes (CNTs) lie in the area of biomedical science and biotechnology [1]. For example, CNTs have been proposed as a nanoscale vehicle for drug delivery [1-3] and formed the basis of the development of a new in situ human organ pressure monitor [4-5]. A CNT is small enough to cross the cell membrane in a harmless manner and enter the nucleus of the cell, while the cell does not recognize the nanotube as an unfriendly intruder [6]. Therefore, a CNT can be used effectively as a vehicle to deliver drugs to the targeted sick cell (such as a cancer cell) with unprecedented accuracy and efficiency. One possible way to direct the motion of CNTs for drug delivery is CNT spearing, based on the penetration of nickelembedded CNTs into cell membranes driven by a magnetic field [7].

To fulfill the drug delivery process, drug molecules may be attached to a CNT and then released after being carried by the CNT to the targeted areas. A number of studies have demonstrated the feasibility of attaching molecules to a CNT via surface functionalization $[2,8,9]$. Drug molecules may also be placed inside a CNT for delivery. Molecular dynamics (MD) simulations showed that a DNA molecule could be spontaneously encapsulated into a CNT in aqueous solution [10]. Furthermore, transporting and nanopumping atoms

Address correspondence to fliu@eng.utah.edu 
and molecules inside a CNT have been shown using a variety of different actuation mechanisms, driven by thermal [11], electrical [3, 12], optical [13], or mechanical [14, 15] effects.

Because of the attractive interactions, such as van der Waals attraction, between a guest molecule and a CNT, the molecule would prefer to stay inside the CNT in the ground state [10]. In general, there is an energy barrier preventing an encapsulated molecule inside a CNT from coming out of the CNT [16]. Therefore, one needs to search for an effective actuation mechanism to eject the molecule out of the single-walled carbon nanotube (SWNT) for drug delivery. An interesting domino process in SWNTs was recently proposed by Chang [17]. However, such a domino process is only applicable to large tubes when their ground state has a collapsed shape and the circular tube is metastable. Here, we propose a different actuation mechanism via mechanical wave propagation, which is applicable to small tubes. So, these two methods are complementary to each other working with different driving mechanisms and in different ranges of tube diameters. We point out that practically, our proposal of working with small tubes may have two advantages. First, most synthesis methods produce SWNTs of small diameters while larger ones are less common. Second, large SWNTs often take the ground-state collapsed shape [18] rather than the metastable circular shape, preempting use of the domino process.

Specifically, we propose to actuate mechanical wave propagation in an SWNT by two oscillating tips [19]. Using MD simulations that include the tip effect, we demonstrate mechanical nanopumping of a $C_{20}$ molecule via an SWNT. Two tips placed in one end of the SWNT oscillate in such a way as to generate sustained propagating mechanical waves in the tube, which drive and accelerate the molecule inside until ejecting it out from the other end of the tube. We investigate the optimal tip oscillation conditions (i.e., the oscillation frequency and magnitude) in order to find the highest nanopumping efficiency that produces the largest molecule acceleration and ejection speed.

Our MD simulation setup and procedure are shown in Fig. 1(a). A $C_{20}$ molecule encapsulated inside a $(13,0)$ SWNT was chosen as a model system, and was shown to be a stable system based on their respective sizes [16, 20]. Two tips are placed at one end of the tube, which oscillate as a mechanical actuator to generate mechanical wave propagation in the tube as studied previously for the free tubes without the encapsulated molecule [19]. The carboncarbon interactions in the $(13,0)$ SWNT and $C_{20}$ molecule were described by the Tersoff bondorder [21] potential, and the $\mathrm{C}_{20}$-tube interaction was described by a Lennard-Jones potential [22]. We used the Hoover thermostat to perform the MD simulations in the $(N, V, T)$ ensemble at $300 \mathrm{~K}$ and a 5 th order predictor-corrector algorithm to integrate the equation of motion. The time step was set at $0.2 \mathrm{fs}$ with a total simulation time of up to 20 ps. The system was first brought into equilibrium at $300 \mathrm{~K}$, before applying the tip actuation for the nanopumping process.

The static energy barrier for a $\mathrm{C}_{20}$ molecule to come out from a $(13,0)$ SWNT is calculated to be 1.48 $\mathrm{eV}$. Therefore, the $\mathrm{C}_{20}$ has to be accelerated inside the $(13,0)$ tube to gain sufficient kinetic energy to overcome this barrier, in order to realize the

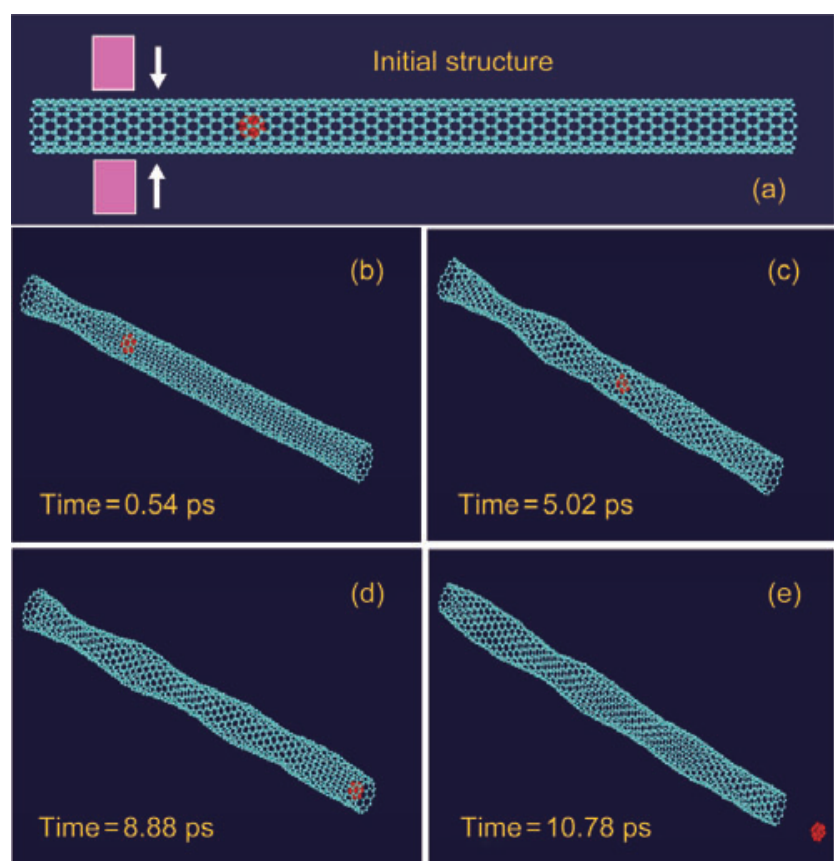

Figure 1 (a) Illustration of simulation setup for nanopumping. (b) to (e) Snapshots of a successful nanopumping process of $C_{20}$ via a $(13,0)$ SWNT. Tip oscillation magnitude is $2.083 \AA$. Tip oscillation frequency is $1 \mathrm{THz}$. Tube length is $136 \AA$ 
nanopumping process. Our previous studies showed that sustainable mechanical wave propagation can be generated by a two-tip actuator [19]. For a $(13,0)$ tube, the mechanical wave velocity can reach as high as $5000 \mathrm{~m} / \mathrm{s}$. Now, in the presence of a $C_{20}$ molecule, as the tip-actuated mechanical wave meets the molecule, the locally deformed (compressed) tube wall will repel the $C_{20}$ driving (squeezing) it to move along the wave propagation direction. The idea is to find optimal tip actuation conditions that will lead to successful nanopumping (accelerating and ejecting) of the $C_{20}$ molecule by the $(13,0)$ SWNT.

Figures 1(b)-(e) show an example of the simulation of a successful nanopumping process. The details of the whole process can be viewed online in the video in the Electronic Supplementary Material (ESM). At the beginning, the $\mathrm{C}_{20}$ is loaded inside next to the two tips placed at one end of the $(13,0)$ tube. As the tips oscillate, the tip-actuated mechanical wave starts to drive the $C_{20}$ to move towards the right due to the repulsive force between the molecule and the deformed tube (Fig. 1(b)). The mechanical waves are generated gradually and sustained throughout the simulation, and accelerate the molecule causing it to move continuously in the direction of the mechanical wave propagation (Fig. 1(c)). After $\sim 9$ ps, the $\mathrm{C}_{20}$ approaches the other free end of the tube (Fig. 1(d)); at $\sim 10 \mathrm{ps}$, the $\mathrm{C}_{20}$ is finally ejected out of the tube (Fig. 1(e)), completing the nanopumping process.

The nanopumping process consists of two key steps of transporting (i.e., accelerating) the molecule inside the tube and ejecting the molecule out of the tube. These can be analyzed by examining the molecule axial velocity as a function of time and tip position as shown in Fig. 2. It is found that most acceleration of $\mathrm{C}_{20}$ occurs in the first 2.0 ps over a short distance of $\sim 6-7 \AA$ (Fig. 2), during which the axial velocity of $\mathrm{C}_{20}$ increases from 0 to $\sim 1100 \mathrm{~m} / \mathrm{s}$ corresponding to a very large acceleration of $\sim 5.5 \times 10^{14}$ $\mathrm{m} / \mathrm{s}^{2}$. Then, the $C_{20}$ continues to accelerate at a slower rate for the next 2.5 ps over a distance of $\sim 25 \AA$ reaching the maximum axial velocity of $1500 \mathrm{~m} / \mathrm{s}$ at an average acceleration of $\sim 1.6 \times 10^{14} \mathrm{~m} / \mathrm{s}^{2}$. After that the $C_{20}$ gradually slows down, decelerating uniformly to an axial velocity of $\sim 1000 \mathrm{~m} / \mathrm{s}$ upon reaching the right-hand open end of the tube before being ejected out of the tube. During the ejection process, as seen at the end of the tube (marked with a vertical dashed line in Fig. 2), the $C_{20}$ velocity first increases slightly as it comes out of the tube but remains bonded with the tube atoms at the end, and then decreases rapidly after breaking the bonds with the tube atoms to become free.

Despite the slow-down of $C_{20}$ as it reaches the right-hand end of the tube, it still possesses sufficient kinetic energy to overcome the potential energy barrier to moving out of the tube. The ejection speed, i.e., the instantaneous axial velocity of $\mathrm{C}_{20}$ leaving the right-hand end of the tube is $\sim 1200 \mathrm{~m} / \mathrm{s}$. As soon as the molecule is ejected out from the tube, its axial velocity decreases rapidly in the vacuum. MD simulations showed that in addition to the lateral translational motion driven by the mechanical wave, the encapsulated $\mathrm{C}_{20}$ also has a rotational motion, i.e., it simultaneously rotates as it translates from left to right. So, through the interaction between $\mathrm{C}_{20}$ and the tube wall, the tube mechanical wave transfers the energy into the $\mathrm{C}_{20}$ in two kinetic energy forms of translational motion and rotational motion. The fluctuation of $\mathrm{C}_{20}$ axial velocity, i.e., translational kinetic energy, as shown in Fig. 2, is largely caused by exchange of these two forms of kinetic energy.

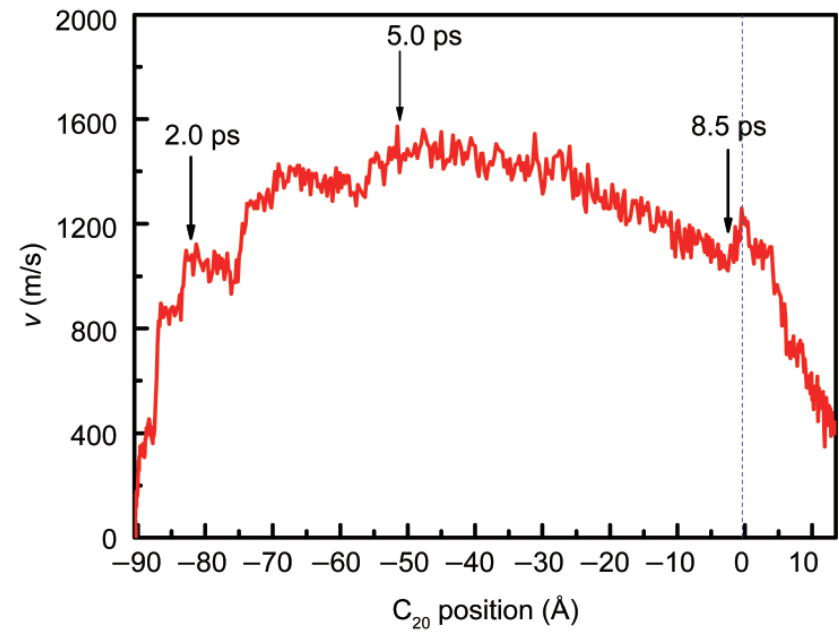

Figure 2 Axial velocity of $C_{20}$ as a function of its position inside the tube during the nanopumping process corresponding to Fig. 1. The right-hand end of the tube is set to be the position zero (vertical dashed line). The three arrows mark the time at different positions, indicating three stages of acceleration and deceleration of $C_{20}$ inside the tube 
When the molecule rotates rapidly, it will slow down its translational motion along the tube axis. Thus, it is desirable to minimize the rotational motion in order to increase nanopumping efficiency. The rotational motion of $\mathrm{C}_{20}$ is possibly caused by its spherical shape giving a small momentum of inertia for rotation. We expect that a non-spherical molecule would undergo less rotation.

A focus of our study is to investigate the efficiency of nanopumping driven by wave propagation and thus find the optimal tip actuation conditions for nanopumping. Most importantly, we found that not all the nanopumping processes are successful and the efficiency of nanopumping depends strongly on the form of mechanical waves generated by the tip actuator, which in turn depends on the tip oscillation conditions. The encapsulated $\mathrm{C}_{20}$ can be pumped out of the $(13,0)$ tube when the two tips oscillate with a correlation between the tip oscillation frequency and magnitude that generates a sustainable traveling wave corresponding to the similar condition for generating the quasi steady-state standing waves in a free $(13,0)$ nanotube without molecule encapsulation [19].

For a given tube length, it has been shown that a smaller tip oscillation magnitude combined with a higher tip oscillation frequency can sustain a more steady mechanical wave in free SWNTs [19]. This indicates that a more stable mechanical wave is desirable for accelerating the molecule inside the tube. Moreover, MD simulations showed that the barrier at the right-hand end of the tube is dynamically changed in the presence of a mechanical wave and is different from the original theoretical barrier for a stationary tube, and furthermore it is slightly different for different waves in every nanopumping process. Therefore, the overall nanopumping process is decided by the optimization of tip actuations to generate a stable sustainable mechanical wave that will both accelerate the molecule inside the tube for transportation and overcome the dynamic barrier at the end of tube for ejecting the molecule.

When the tip actuator does not oscillate according to the desired correlated conditions, our simulations show that the nanopumping process may fail to eject the $\mathrm{C}_{20}$ molecule from $(13,0) \mathrm{SWNT}$, as shown in Fig. 3. This is because away from the optimal tip oscillation magnitude or frequency, the tip actuation cannot generate a steady mechanical wave, but instead the wave dies out with time, as in a free tube [19]. Furthermore, a non-steady irregular wave tends to induce large rotation of $\mathrm{C}_{20}$ causing the whole tube to bend at the same time (Fig. 3). These actions all dissipate a lot of energy and reduce the acceleration of $\mathrm{C}_{20}$ in the axial direction. Whenever these two effects are significant, the nanopumping process fails.

We can examine the effectiveness of nanopumping process by analyzing the energy transfer process in terms of kinetic energy partition between the translational and rotational motion. Figure 4 shows a comparison of the kinetic energy partition between a successful and a failed nanopumping process. In a successful nanopumping process (Fig. 4(a)), the tube mechanical wave energy is transferred into the $\mathrm{C}_{20}$ mostly in the form of translational kinetic energy, while the gain in rotational kinetic energy is very small. Consequently, the $\mathrm{C}_{20}$ gains up to $\sim 2$ $\mathrm{eV}$ of translational kinetic energy in the first few ps

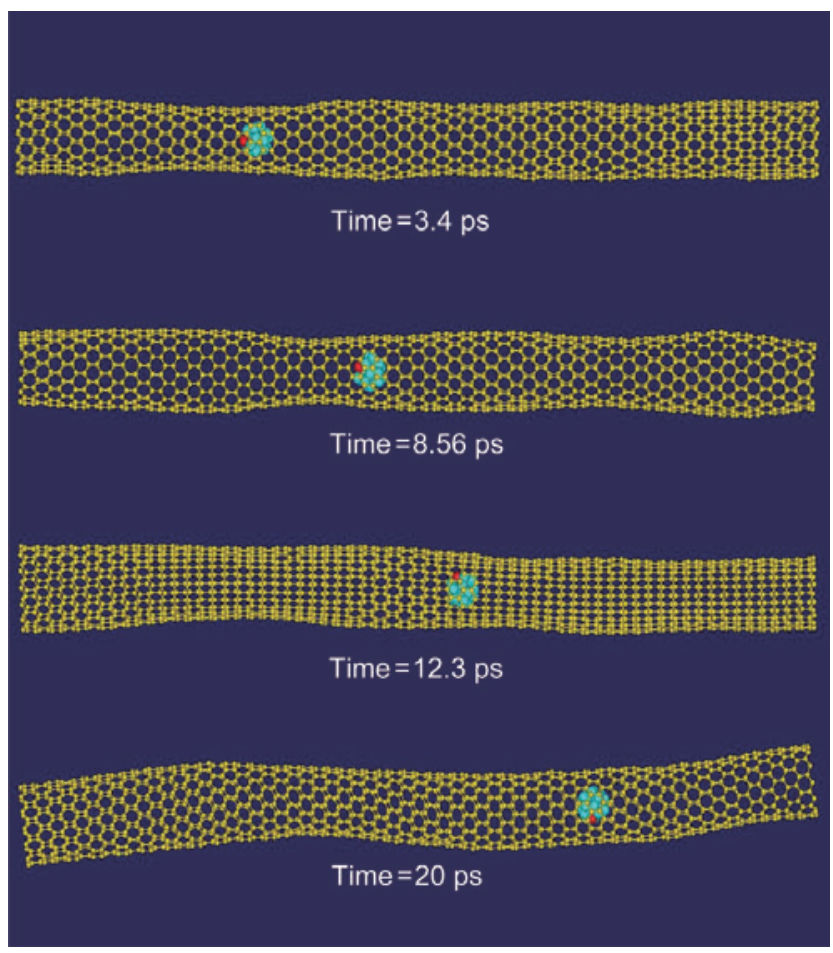

Figure 3 Snapshots of a failed nanopumping process of $C_{20}$ via a $(13,0)$ SWNT. One atom in $C_{20}$ is marked red to show the rotational motion of $C_{20}$. Tip oscillation magnitude is $1.583 \AA$. Tip oscillation frequency is $0.25 \mathrm{THz}$. Tube length is $136 \AA$ 
and is then ejected out at $\sim 9.8$ ps. In contrast, in a failed nanopumping process (Fig. 4(b)), a significant fraction of the tube mechanical wave energy is transferred into the $\mathrm{C}_{20}$ rotational kinetic energy. Consequently, the $\mathrm{C}_{20}$ gains much less translational kinetic energy (less than $\sim 0.45 \mathrm{eV}$ ), which is not enough for ejection of the molecule out of the tube even after 20 ps of simulation.

Because successful nanopumping can only be achieved with certain correlated tip oscillation magnitudes and frequencies, we have carried out extensive MD simulations to determine the suitable range of the tip oscillation magnitudes and frequencies leading to successful nanopumping, and constructed a phase diagram as shown in Fig. 5. It is found that the range of tip oscillation magnitudes for successful nanopumping is from 0.583 to $3.583 \AA$ using $(13,0)$ SWNT. If the tip oscillation magnitude is too small $(<0.583 \AA)$, the mechanical wave does not generate enough force and energy to drive the molecule. If the tip oscillation magnitude is too large (>3.583 $\AA$ ), it causes the upper portion of the tube to form bonds with the lower part in the compressed regions between the two tips leading to breakdown of actuation.

At any given tip oscillation magnitude between $0.583 \AA$ and $3.583 \AA$, the desired tip frequency for successful nanopumping is different. In general, a larger oscillation magnitude requires lower frequencies. For example, when the tip oscillation magnitude is $3.083 \AA$, the range of successful tip frequencies is $0.15-0.71 \mathrm{THz}$, as shown in Fig. 5. If the frequency is lower than $0.15 \mathrm{THz}, \mathrm{C}_{20}$ exhibits large rotational motion and the tube begins to bend. Consequently, the $C_{20}$ will either fail to reach the other end of the tube, or fail to come out of the tube even if it reaches the end of the tube. If the frequency is higher than $0.71 \mathrm{THz}$, no obvious mechanical wave can be generated in the tube, due to the lack of time for the tube to respond to the tip oscillation.

Different forms of mechanical wave in $(13,0)$ SWNT drive the encapsulated $C_{20}$ molecule with different efficiencies. In particular, the acceleration of the $\mathrm{C}_{20}$ molecule inside the tube increases with the increasing tip frequency, but it can decrease if the frequency is too high. The reason is that the higher

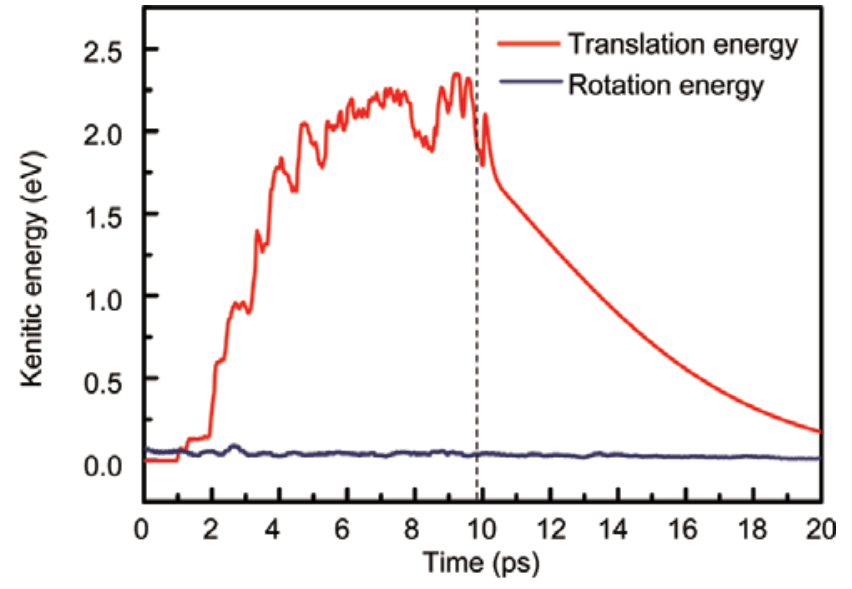

(a)

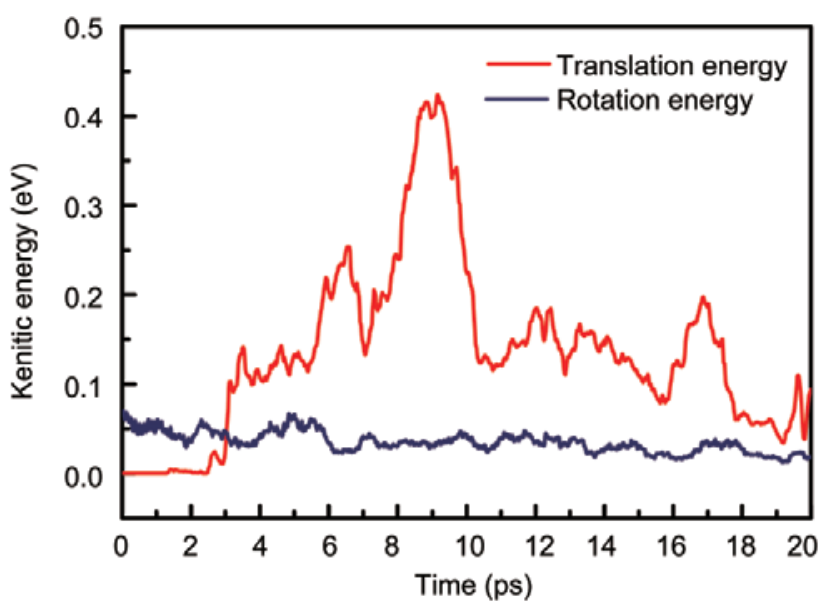

(b)

Figure 4 Comparison of kinetic energy partition between the translational and rotational motion of $C_{20}$ in (a) a successful pumping process versus in (b) a failed pumping process

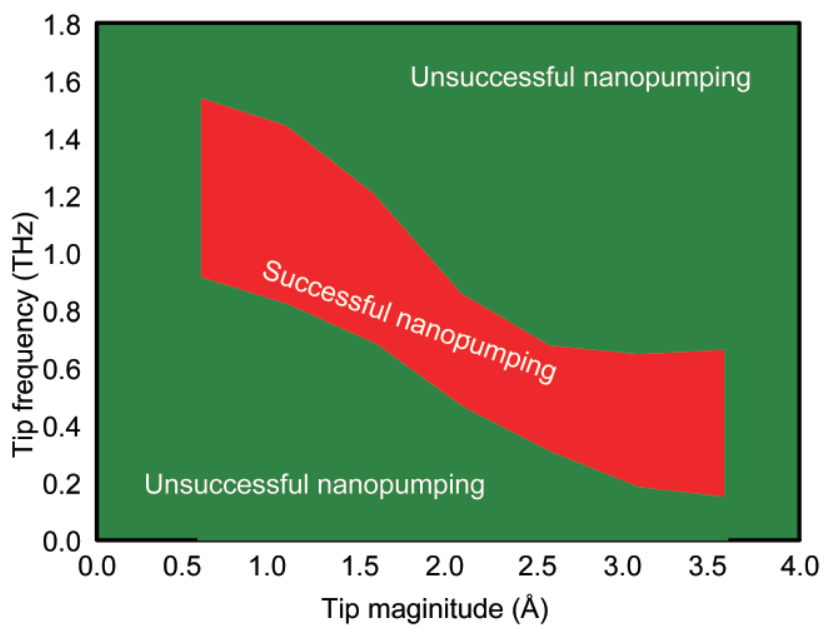

Figure 5 Phase diagram that defines the range of tip oscillation magnitudes and frequencies for successful nanopumping 
driving tip frequency has a higher "horsepower" injecting more energy per unit time into the tube. But, if the frequency is too high, the tube cannot mechanically respond to the tip oscillation. On the other hand, for the successful nanopumping processes, the molecule ejection velocities are quite different under different tip conditions (see Table S-1 in the ESM where a few examples of different accelerations and ejection speeds of $\mathrm{C}_{20}$ resulting from different tip oscillation magnitudes and frequencies are listed). This is because the energy transfer process between the molecule and the SWNT varies for different mechanical waves generated in the tube, leading to different molecular transport processes and ejection behaviors. The maximum acceleration and ejection velocities of $C_{20}$ via a $(13,0)$ SWNT we achieved are $3.44 \times 10^{14} \mathrm{~m} / \mathrm{s}^{2}$ and $2176 \mathrm{~m} / \mathrm{s}$, respectively.

In summary, using MD simulations we have demonstrated the feasibility of using SWNTs for nanopumping molecules via mechanical wave propagation driven by an oscillating tip actuator. We found that there exists an optimal tip oscillation condition for the nanopumping process, which corresponds to the generation of quasi steadystate wave propagation in the tube so that the tube mechanical wave energy is transferred mostly to the molecular translational motion rather than the rotational motion. The nanopumping is usually more efficient using smaller tip oscillation magnitudes and higher frequencies. Our proposed nanopumping process using small tubes is complementary to the domino process using large tubes [17]. These findings may help develop a useful approach for drug delivery using SWNTs.

\section{Acknowledgements}

We thank Center for High Performance Computing at the University of Utah for providing the computing resources. This work is supported by Department of Energy - Basic Energy Science program. In addition, M. C. thanks the China Scholarship Council and D. X. thanks the Natural Science Foundation of China (Nos. 50410179 and 50772068) for their support.
Electronic Supplementary Material: A video and table giving details of the nanopumping process are available in the online version of this article at http://dx.doi.org/10.1007/s12274-009-9096-6 and are accessible free of charge.

\section{References}

[1] Martin, C. R.; Kohli, P. The emerging field of nanotube biotechnology. Nat. Rev. Drug Discov. 2003, 2, 29-37.

[2] Kam, N. W. S.; Dai, H. J. Carbon nanotubes as intracellular protein transporters: Generality and biological functionality. J. Am. Chem. Soc. 2005, 127, 6021-6026.

[3] Svensson, K.; Olin, H.; Olsson, E. Nanopipettes for metal transport. Phys. Rev. Lett. 2004, 93, 145901.

[4] Wu, J.; Zang, J.; Larade, B.; Guo, H.; Gong, X. G.; Liu, F. Computational design of carbon nanotube electromechanical pressure sensors. Phys. Rev. B 2004, 69, 153406.

[5] Liu, F. In situ pressure monitor and associated methods. Patent, Pub. No. WO/2008/127797, Oct. 23,2008; International Application No. PCT/US2008/055525, Feb. 29, 2008

[6] Pantarotto, D.; Briand, J. -P.; Prato, M.; Bianco, A. Translocation of bioactive peptides across cell membranes by carbon nanotubes. Chem. Commun. 2004, 1, 16-17.

[7] Cai, D.; Mataraza, J. M.; Qin, Z. -H.; Huang, Z.; Huang, J.; Chiles, T. C.; Carnahan, D.; Kempa, K.; Ren, Z. Highly efficient molecular delivery into mammalian cells using carbon nanotube spearing. Nat. Methods 2005, 2, 449454.

[8] Smith, B. W.; Monthioux, M.; Luzzi, D. E. Encapsulated C-60 in carbon nanotubes. Nature 1998, 396, 323-324.

[9] Hummer, G.; Rasalah, J. C.; Noworyta, J. P. Water conduction through the hydrophobic channel of a carbon nanotube. Nature 2001, 414, 188-190.

[10] Gao, H.; Kong, Y.; Cui, D.; Ozkan, C. S. Spontaneous insertion of DNA oligonucleotides into carbon nanotubes. Nano. Lett. 2003, 3, 471-473.

[11] Longhurst, M. J.; Quirke, N. Temperature-driven pumping of fluid through single-walled carbon nanotubes. Nano. Lett. 2007, 7, 3324-3328.

[12] Dai, Y.; Tang, C.; Guo, W. Simulation studies of a "nanogun" based on carbon nanotubes. Nano Res. 
2008, 1, 176.

[13] Král, P.; Tománek, D. Laser-driven atomic pump. Phys. Rev. Lett. 1999, 82, 5373-5376.

[14] Insepov, Z.; Wolf, D.; Hassanein, A. Nanopumping using carbon nanotubes. Nano. Lett. 2006, 6, 1893.

[15] Wang, Q. Atomic transportation via carbon nanotubes. Nano. Lett. 2009, 9, 245-249.

[16] Ulbricht, H.; Moos, G.; Hertel, T. Interaction of $C_{60}$ with carbon nanotubes and graphite. Phys. Rev. Lett. 2003, 90, 095501.

[17] Chang, T. Dominoes in carbon nanotubes. Phys. Rev. Lett. 2008, 101, 175501.

[18] Chopra, N. G.; Benedict, L. X.; Crespi, V. H.; Cohen, M. L.; Louie, S. G.; Zettl, A. Fully collapsed carbon nanotubes. Nature 1995, 377, 135-138.
[19] Chen, M.; Zang, J.; Xiao, D. Q.; Liu, F. Mechanical wave propagation in carbon nanotubes driven by an oscillating tip actuator. J. Appl. Phys. 2009, 105, 026102.

[20] Okada, S.; Saito, S.; Oshiyama, A. Energetics and electronic structures of encapsulated C-60 in a carbon nanotube. Phys. Rev. Lett. 2001, 86, 3835-3838.

[21] Tersoff, J. Sample-dependent resolution in scanning tunneling microscopy. Phys. Rev. B 1989, 39, 10521057.

[22] Rappé, A. K.; Casewit, C. J.; Colwell, K. S.; Goddard III, W. A.; Skiff, W. M. UFF, a full periodic-table forcefield for molecular mechanics and molecular-dynamics simulations. J. Am. Chem. Soc. 1992, 114, 1002410035. 